\title{
Revisão de literatura e relato de caso de fístula arteriovenosa craniana cutânea pós-traumática
}

\author{
Jefferson Rosi Junior', Eberval Gadelha de Figueiredo², \\ Lin Tchia Yeng ${ }^{3}$, Manoel Jacobsen Teixeira ${ }^{4}$ \\ Divisão de Clínica Neurocirúrgica do Hospital das Clínicas da Faculdade de Medicina da Universidade de São Paulo \\ (HC-FMUSP).
}

\section{RESUMO}

Os autores descrevem o caso de um doente de 34 anos que, após agressão sofrida em 2008, detectou o surgimento de tumoração no mesmo local em que sofrera a agressão, onde surgiu uma fístula arteriovenosa cutânea pós-traumática, documentada por meio de angiografia digital. Tanto o doente quanto a equipe médica optaram por conduta não cirúrgica, e o doente segue assintomático no seguimento ambulatorial, sem modificação da fístula desde seu diagnóstico.

\section{PALAVRAS-CHAVE}

Aneurisma, fístula arteriovenosa, angiografia cerebral, embolização terapêutica, traumatismos craniocerebrais.

\section{ABSTRACT}

Post traumatic cutaneous cranial fístula: case report and literature review

The authors describe a 34 years old men with cutaneous vascular disorder after a cranial injury suffered three months ago. Subsidiary investigation by angiography showed a cutaneous arteriovenous malformation. The men asymptomatic, have a non-surgical conduct and he is very well after three years.

\section{KEYWORDS}

Aneurysm, arteriovenous fistula, cerebral angiography, embolization terapeuthics, craniocerebral trauma.

1 Neurocirurgião do Hospital das Clínicas da Faculdade de Medicina da Universidade de São Paulo (HC-FMUSP), São Paulo, SP, Brasil.

2 Chefe do Grupo de Neurovascular e Supervisor da Divisão de Neurocirurgia do HC-FMUSP, São Paulo, SP, Brasil.

3 Chefe do Ambulatório de Dor em Fisiatria do HC-FMUSP, São Paulo, SP, Brasil.

4 Professor titular de Neurocirurgia do HC-FMUSP, São Paulo, SP, Brasil. 


\section{Introdução}

Aneurisma cirsóideo, aneurisma serpentinum e angioma plexiforme são sinonímias da fístula arteriovenosa cutânea, que consiste em má-formação vascular do couro cabeludo drenada por dilatadas e tortuosas veias.

As fístulas arteriovenosas cutâneas podem ter origem congênita ou traumática, e os homens são mais acometidos por elas. ${ }^{1,2}$

Os autores descrevem um doente com fístula arteriovenosa cutânea traumática (FAVCT) e discutem as possibilidades de abordagem terapêutica dessa condição.

\section{Relato de caso}

Paciente, 34 anos, sofreu agressão por paulada desferida contra sua fronte em novembro de 2008. Três meses após observou o surgimento de pequeno tumor na região e resolveu procurar atendimento especializado.

Sem déficits neurológicos e sem quaisquer outras queixas de saúde, apresentava, em fevereiro de 2009, somente o pequeno tumor na fronte, com frêmito palpável e sensação semelhante à do palpar de uma fístula arteriovenosa criada em membro superior para hemodiálise.

Realizou tomografia computadorizada cranioencefálica sem alterações.

Realizou ultrassonografia com Doppler da região paramediana frontal direita, que detectou presença de fístula arteriovenosa no plano subcutâneo, junto à tábua óssea craniana externa, com extensão medida de $2,8 \mathrm{~cm}$, sem comprometimento de planos mais profundos, e angiografia digital, como apresentado nas figuras 1, 2 e 3, demonstrando opacidade precoce de veia cefálica extracraniana por laceração de ramo subcutâneo da região frontal paramediana direita, que se opacifica pelo ramo frontal da artéria oftálmica direita e por anastomoses pelo homônimo oposto e pelas artérias temporais superficiais, com drenagem efetuada em direção às veias faciais.

Avaliada a possibilidade de extirpação cirúrgica da lesão, o doente se negou a realizar o procedimento, visto que não desejava uma cicatriz na região, bem como não aderiu à ideia de embolização por temer haver necrose do couro cabeludo.

Desse modo, permanece sendo visto no consultório de neurocirurgia vascular anualmente, assintomático até o momento e sem haver aumento da lesão (Figuras $1,2$ e 3$)$.

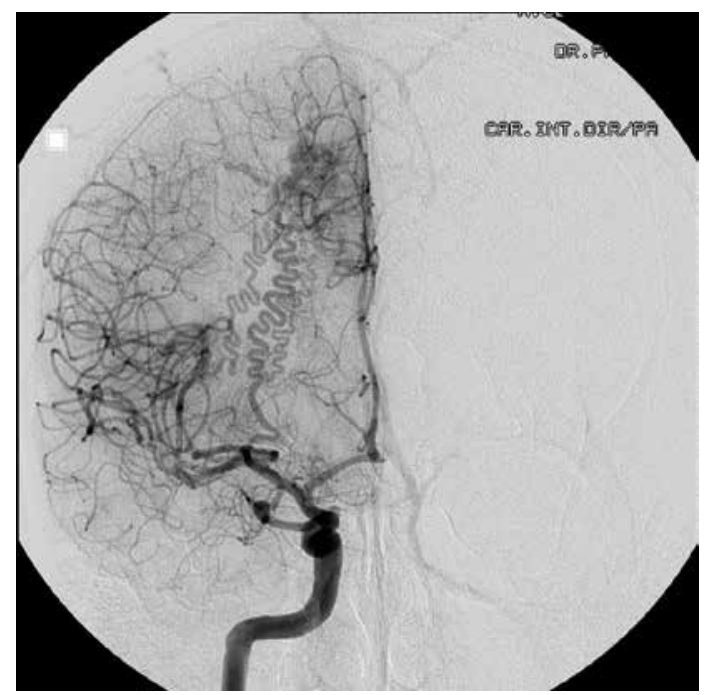

Figura 1 - Angiografia de artéria carótida interna direita em anteroposterior demonstrando FAVCT.

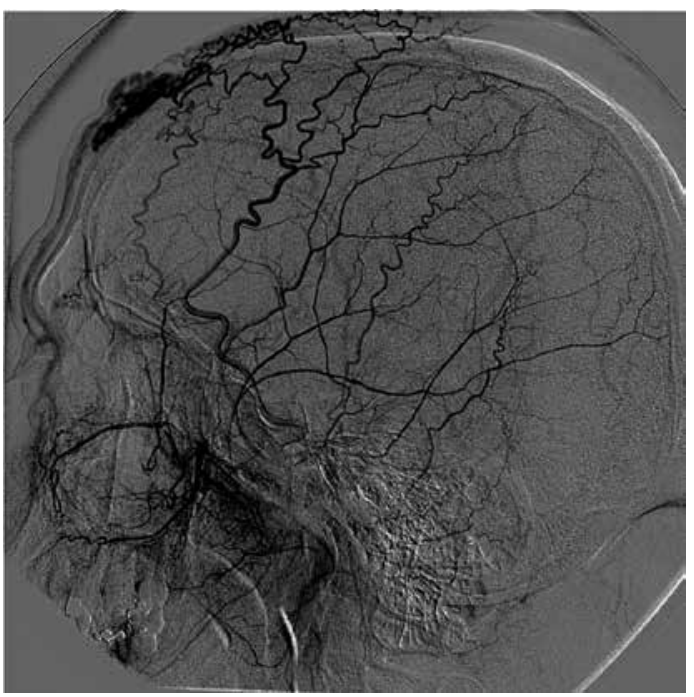

Figura 2 - Angiografia em perfil, já na fase venosa, mostra persistência do meio de contraste na FAVCT.

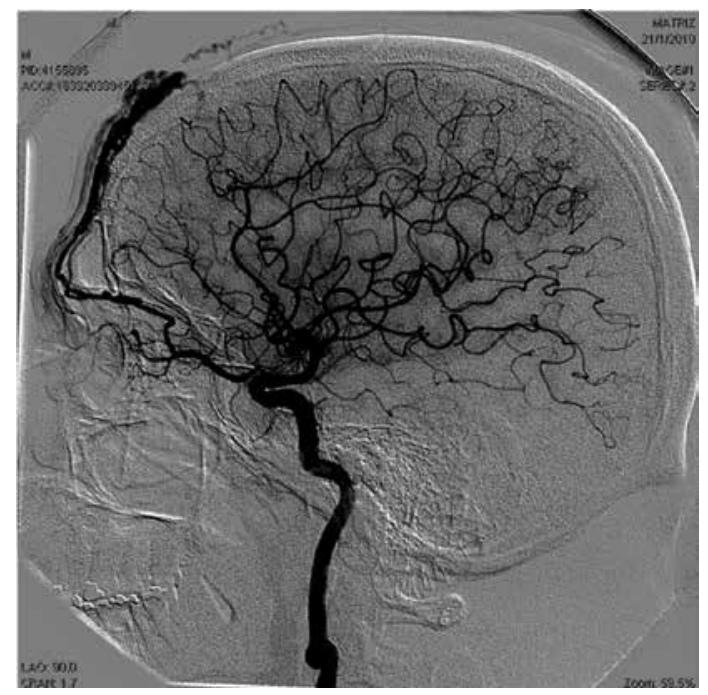

Figura 3 - Angiografia em perfil da artéria carótida mostra a FAVCT. 


\section{Discussão}

As FAVCT costumam acometer com semelhante frequência as regiões frontal, temporal e parietal, e os doentes com FAVCT queixam-se da existência de massa pulsátil e deformante no couro cabeludo, alguns se queixando de cefaleia também.

O exame físico permite a palpação de tumor pulsátil, de frêmito e eventualmente a ausculta de sopro.

Uma vez feita a suspeita clínica de FAVCT, torna-se imperativa a realização de angiografia, que fornece informações sobre localização, tamanho, número de artérias nutridoras, padrão de drenagem venosa, além de afastar uma eventual comunicação da FAVCT com vasos intracranianos. ${ }^{3-8}$

Tomografia computadorizada cranioencefálica ou ressonância do encéfalo também devem ser realizadas com objetivo de afastar a coexistência de outras doenças intracranianas. ${ }^{9,10}$

A ultrassonografia com Doppler permite estudo dinâmico e não invasivo da FAVCT, sendo, assim, de grande praticidade e utilidade. ${ }^{1}$

A perda de continuidade na parede do vaso ocorrida no traumatismo e o extravasamento do sangue podem levar a uma reparação normal do vaso, ou eventualmente o surgimento de uma FAVCT, ${ }^{1,11}$ o que pode ocorrer tanto por conta da reparação anormal dos vasos quanto por uma proliferação de brotos endoteliais a partir da vasa vasorum dos vasos lesados pelo traumatismo. ${ }^{9,11}$

As FAVCT são conexões de ramos periféricos da artéria carótida externos com veias no couro cabeludo. ${ }^{6,12-14}$ Embora o couro cabeludo se envolva na maioria dos traumatismos cranioencefálicos (TCE), o surgimento de FAVCT é uma raridade, e os homens tradicionalmente mais expostos ao TCE são mais acometidos.

Sendo o transplante de cabelo e as próprias incisões para craniotomia formas de traumatismo sobre o couro cabeludo, as FAVCT potencialmente podem ter origem também nessas condições, como já descrito pela literatura. ${ }^{1,3,6,7,12-21}$

O tratamento da FAVCT pode ser realizado por meio de excisão cirúrgica, ligadura dos vasos nutridores da FAVCT, embolização e eletrotrombose. Há necessidade, na cirurgia, de excisão completa da fístula por ligadura de todos os ramos que a nutrem, pois do contrário há a possibilidade de recidiva. ${ }^{3,4,7,17-19,22-24}$

Outras complicações em potencial da cirurgia são necrose do couro cabeludo e infecção. ${ }^{4,6,16}$

A terapia endovascular é alternativa ao tratamento cirúrgico clássico das FAVCT, podendo ser usada como método único ou em combinação com a cirurgia clássica. A embolização, como adjuvante à terapêutica cirúrgica, reduz a perda sanguínea na cirurgia. ${ }^{3,9,15,25,26}$
As FAVCT são lesões de alto débito, nutridas pelas artérias temporal superficial, occipital e meníngea média. A embolização por punção direta pode ser realizada sob sedação e anestesia local por injeção de N-butil-cianoacrilato (NBCA) misturado a lipiodol. Deve-se tomar cuidado, pois há a possibilidade de embolia do material para a circulação pulmonar, fato evitado por compressão mecânica das veias de drenagem da FAVCT através de anel metálico.

Embolização transarterial realizada por microcateterismo femoral com cateter-guia na carótida externa, sendo utilizado também o NBCA misturado a lipiodol, constitui-se em outra modalidade terapêutica via endovascular, em que micromolas e álcool absoluto também podem ser usados para embolizar. ${ }^{10,27}$

O uso de cateter-guia com balão oclusor para controle de fluxo proximal é fundamental para evitar embolias a distância, se escolhida essa técnica. ${ }^{3-5,7,8,11,16,17,20}$

As FAVCT devem ter diagnóstico diferencial com o do sinus pericranii, que consiste em coleção de vasos sanguíneos venosos aderidos à tábua externa do crânio, comunicados com algum seio venoso intracraniano por meio de veias diploicas. ${ }^{8.10,25,26,28}$

\section{Conflito de interesses}

Os autores declaram inexistência de conflito de interesses na realização deste trabalho.

\section{Referências}

1. Aranda G, González R, Preto R, de Górriz L, Cobhan $M$, Lombardo M. Arteriovenous fistula of the superficial temporal artery. Rev Med Panama. 1991;16(3):211-4.

2. Badejo L, Rockwood P. Traumatic arteriovenous fistula of the scalp. Case report. J Neurosurg. 1987;66(5):773-4.

3. Barnwell SL, Halbach VV, Dowd CF, Higashida RT, Hieshima GB. Endovascular treatment of scalp arteriovenous fistulas associated with a large varix. Radiology. 1989;173(2):533-9.

4. Bitoh S, Hasegawa H, Fujiwara M, Nakata M. Traumatic arteriovenous fistula between the middle meningeal artery and cortical vein. Surg Neurol. 1980;14(5):355-8.

5. Duncan IC, Fourie PA. Circumferential flow reduction during percutaneous embolotherapy of extracranial vascular malformations: the "cookie-cutter" technique. AJNR Am J Neuroradiol. 2003;24(7):1453-5.

6. Fisher-Jeffes ND, Domingo Z, Madden M, de Villiers JC. Arteriovenous malformations of the scalp. Neurosurgery. 1995;36(4):656-60.

7. Jimenez JP, Goree JA. Traumatic arteriovenous fistula of the superficial temporal artery. Report of a case. Am J Roentgenol Radium Ther Nucl Med. 1969;106(2):279-81. 
8. Vinas FC, Valenzuela S, Zuleta A. Literature review: sinus pericranii. Neurol Res. 1994;16(6):471-4.

9. Gurkanlar D, Gonul M, Solmaz I, Gonul E. Cirsoid aneurysms of the scalp. Neurosurg Rev. 2006;29(3):208-12.

10. Lanzino G, Passacantilli E, Lemole GM Jr, McDougall C, Spetzler RF. Scalp arteriovenous malformation draining into the superior sagittal sinus associated with an intracranial arteriovenous malformation: just a coincidence? Case report. Neurosurgery. 2003;52(2):440-3.

11. Han MH, Seong SO, Kim HD, Chang KH, Yeon KM, Han MC. Craniofacial arteriovenous malformation: preoperative embolization with direct puncture and injection of n-butyl cyanoacrylate. Radiology. 1999;211(3):661-6.

12. LaHaye PA, Lawner PM. Cortical arteriovenous fistula associated with skull fracture. Case report. J Neurosurg. 1984;60(1):192-5.

13. Rosi Junior J. Neurônio: perguntas e respostas em neurocirurgia. São Paulo: Scortecci; 2011.

14. Souder DE, Bercaw BL. Arteriovenous fistula secondary to hair transplantation. N Engl J Med. 1970;283(9):473-4.

15. Kasdon DL, Altemus LR, Stein BM. Embolization of a traumatic arteriovenous fistula of the scalp with radiopaque Gelfoam pledgets. Case report and technical note. J Neurosurg. 1976;44(6):753-6.

16. Khodadad G. Familial cirsoid aneurysm of the scalp. J Neurol Neurosurg Psychiatry. 1971;34(6):664-7.

17. Komatsu Y, Narushima K, Kobayashi E, Nose T, Maki Y. Congenital arteriovenous malformation of the scalp--case report. Neurol Med Chir (Tokyo). 1989;29(3):230-4.

18. Lanzieri CF, Sacher M, Som PM, Haimov M. Arteriovenous fistula after hair transplantation. AJNR Am J Neuroradiol. 1985;6(1):111-2.

19. Morioka T, Nishio S, Hikita T. Traumatic arteriovenous fistulae of the scalp at the area of previous craniotomy. Surg Neurol. 1988;30(5):406-7.
20. Shenoy SN, Raja A. Scalp arteriovenous malformations. Neurol India. 2004;52(4):478-81.

21. Williams LR, Robinson JK, Yao JS. Hair transplantation producing arteriovenous fistulization. Ann Vasc Surg. 1986;1(2):241-3.

22. Mourao GS, Hodes JE, Gobin YP, Casasco A, Aymard A, Merland JJ. Curative treatment of scalp arteriovenous fistulas by direct puncture and embolization with absolute alcohol. Report of three cases. J Neurosurg. 1991;75(4):634-7.

23. Nagasaka S, Fukushima T, Goto K, Ohjimi H, Iwabuchi S, Maehara F. Treatment of scalp arteriovenous malformation. Neurosurgery. 1996;38(4):671-7.

24. Ogawa $\mathrm{Y}$, Inoue K. Electrothrombosis as a treatment of cirsoid angioma in the face and scalp and varicosis of the leg. Plast Reconstr Surg. 1982;70(3):310-8.

25. Madan Rao V, Feig SA. Traumatic arteriovenous fistulae of the scalp. Rev Interam Radiol. 1980;5(1):17-20.

26. Marks MW, Argenta LC, Dingman RO. Traumatic arteriovenous malformation of the external carotid arterial system. Head Neck Surg. 1984;6(6):1054-8.

27. Heilman CB, Kwan ES, Klucznik RP, Cohen AR. Elimination of a cirsoid aneurysm of the scalp by direct percutaneous embolization with thrombogenic coils. Case report. J Neurosurg. 1990;73(2):296-300.

28. Marotta TR, Berenstein A, Zide B. The combined role of embolization and tissue expanders in the management of arteriovenous malformations of the scalp. AJNR Am J Neuroradiol. 1994;15(7):1240-6.

\section{Endereço para correspondência}

Jefferson Rosi Junior

Rua Turiaçu 2237, ap. 71, bloco B, Pompéia

05005-001 - São Paulo, SP, Brasil

Telefone: (11) 9647-8000

E-mail: jefrosijr@gmail.com 